Birmingham Great Park, Bristol Road South, Rubery, Birmingham B45 9BE, S. Geelan Consultant in Forensic Psychiatry, East Midlands Centre for Forensic Mental Health, Arnold Lodge, Cordelia Close, Leicester LE5 OLE

\section{Triage in emergency psychiatry}

Sir: The concept of triage in emergency psychiatry is an interesting one, clearly elucidated by Morrison et al (Psychiatric Bulletin, July 2000, 24, 261-264). Their flow chart elegantly illustrates the process by which cases should be allocated for assessment and one would hope that these considerations would be made in all cases as the number of urgent referrals constantly increases.

However, the final tier of the diagram is perhaps unrepresentative of the resources and manpower available in many departments of psychiatry. There may not be a specialist registrar within the unit and clinical assistants are often part-time, or employed for specific sessions such as day hospital or out-patient clinics. This reduces the staff available to the consultant and senior house officer(s) or the 'on-call' senior house officer. I suspect in practice that the majority of general hospital and accident and emergency referrals are in the first instance dealt with by junior staff, as well as a large proportion of urgent general practitioner referrals. Difficulties may be compounded by manpower shortages and reluctance of locum consultant staff to take on urgent work, other than in a supervisory capacity. In addition, there is rarely a good system in place for monitoring the level of, and response to, emergency referrals.

Although with adequate supervision emergency assessments provide an excellent learning experience for trainees, I feel that their role in the triage and assessment of emergency psychiatric referrals should be clarified and the experience of a senior colleague in providing effective triage utilised to the full.

Sara Smith Senior House Officer in Psychiatry, Kidderminster Hospita

\section{Detoxification from heroin with buprenorphine}

Sir: There are a number of options available for detoxification from heroin, including methadone tapering regimes, dihydrocodeine reduction, lofexidine, and ultra-rapid naltrexone assisted detoxification under general anaesthetic (Sievewright, 2000). Buprenorphine has recently been licenced in the UK for the treatment of opiate dependence and offers an alternative method of withdrawal from heroin; it has proven efficacy for out-patient detoxification (O'Connor et al, 1997) but has been little used in the UK. Here we present the results of a pilot study of 30 consecutive out-patient detoxifications with patients who were using low-dose heroin ( $\mathrm{f} 20$ approximately $0.2 \mathrm{~g}$ daily) using buprenorphine with a standard treatment protocol lasting 7 days.

Of the 30 patients who participated in the study, 15 (50\%) successfully completed the detoxification programme and 15 (50\%) defaulted. Symptom control appears to have been good, with subject showing mild to moderate withdrawal symptoms throughout the detoxification. The consumption of the medication was easily supervised by clinic staff, ensuring good compliance.

This suggests that, for some opiate dependent patients, a standard prescription protocol of buprenorphine can be used effectively for out-patient heroin detoxification with good compliance and good symptom control. However, as of yet there is no evidence to suggest which type of detoxification is the most effective in terms of matching to patient variables, cost, completion rate or symptom control. Leeds Addiction Unit is currently undertaking a randomised control trial of lofexidine $v$. buprenorphine to look at these issues in detail.

O'CONNOR, P. G., CARROL, K. M., SHI, J. M., et al (1997) Three methods of opiate detoxification in primary care setting. Annals of Internal Medicine, 127, 526-530.

SIEVEWRIGHT, N. (1999) CommunityTreatment of Drug Misuse: MoreThan Methadone. Cambridge: Cambridge University Press.

D. Gordon Specialist Registrar， D. S. Raistrick Clinical Director， J. Banbery Associate Specialist, Leeds Addiction Unit, 19 Springfield Mount, Leeds LS2 9NG

\section{Learning disability in psychiatry - the future of services}

Sir: I support what O'Dwyer (Psychiatric Bulletin, July 2000, 24, 247-250) describes of her experiences as a consul tant psychiatrist in learning disability. Her difficulties were recognised by other psychiatrists in the UK. Of fundamental influence on the workload of community teams in learning disability are the number of independent care homes in a catchment area rather than the size of the general population. Poor training and a high turnover of care staff compound the difficulties inherent in the workload that the psychiatrist and the mental health team can expect.

With the move to 'normalisation' of learning disability services since the closure of the institutions and the 'demedicalisation' of care, I believe services have been hijacked by well-meaning professionals and carers who choose not to recognise, or remain ignorant of, mental illness in this group of people. Ultimately they do a disservice to their clients, which in many cases results in eviction from homes because of difficult behaviour or the inappropriate prescription of potent drugs by general practitioners and general psychiatrists. Unfortunately they too can hold society's prejudice towards the learning disabled and thus further stigmatise their patients.

In planning services, the importance of well-resourced mental health teams in learning disability cannot be ignored because society has a lot to gain from the understanding of mental health issues in learning disability, which has the potential for skills and treatments to be generalised to other groups in the population.

Ken Courtenay Specialist Registrar, Department of Psychiatry of Disability, St George's Hospital Medical School, London SW17 ORE

(e-mail: kcourtenay@sghms.ac.uk)

\section{Serotonin syndrome}

Sir: Mir and Taylor's review of serotonin syndrome (Psychiatric Bulletin, December 1999, 23, 742-747) stated that in practice lithium was well tolerated in combination with a selective serotonin reuptake inhibitor (SSRI), but mentioned four individual reports where problems had been experienced. Two of these involved the emergence of serotonin syndrome after the addition of lithium to the treatment regime of a patient already taking an SSRI without side-effects. I would like to add to these a further case seen as an emergency referral to our Affective Disorders Clinic in May 2000.

$\mathrm{Mr} \mathrm{B}$ is a 53 year old professional white male who has been suffering with recurrent depressive episodes for the last 18 months. He had been treated with various antidepressants during this time. At the time of his urgent referral he had been taking paroxetine $60 \mathrm{mg}$ daily for over 3 months, to which lithium $400 \mathrm{mg}$ daily had been added 2 weeks previously.

On presentation Mr B described profound nausea with the addition of five of Sternbach's diagnostic criteria for serotonin syndrome: agitation, myoclonus, shivering, tremor and incoordination. Serum lithium levels at this time were within normal limits. Lithium was discontinued and the paroxetine was reduced slowly over the next 6 weeks. Within a week Mr B's symptoms had improved and on 3 week review he was symptom-free with regard to the serotonin syndrome.

The above case of serotonin syndrome was attributed to the addition of lithium to the SSRI. This was because he was sideeffect-free on treatment with paroxetine and the symptoms developed shortly 